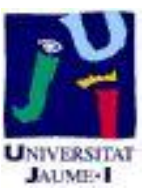

Título artículo / Títol article: Interpretation of cyclic voltammetry measurements of thin semiconductor films for solar fuel applications.

Autores / Autors

Bertoluzzi, Luca ; Badia Bou, Laura ; Fabregat Santiago, Francisco ; Giménez Juliá, Sixto ; Bisquert, Juan

Revista:

The Journal of Physical Chemistry Letters

Versión / Versió:

Post-print

Cita bibliográfica / Cita

BERTOLUZZI, Luca, et al. Interpretation of Cyclic bibliogràfica (ISO 690): Voltammetry Measurements of Thin Semiconductor Films for Solar Fuel Applications. The Journal of Physical Chemistry Letters, 2013, vol. 4, no 8, p. 1334-1339.

url Repositori UJI:

http://hdl.handle.net/10234/89434 


\title{
I. Interpretation of Cyclic Voltammetry Measurements of Thin Semiconductor Films for Solar Fuel Applications
}

\author{
${ }_{3}$ Luca Bertoluzzi, Laura Badia-Bou, Francisco Fabregat-Santiago, Sixto Gimenez, and Juan Bisquert* \\ 4 Photovoltaics and Optoelectronic Devices Group, Departament de Física, Universitat Jaume I, 12071 Castelló, Spain \\ 5 S Supporting Information
}

\begin{abstract}
6 ABSTRACT: A simple model is proposed that allows interpretation of the cyclic voltammetry diagrams obtained experimentally for photoactive semiconductors with surface states or catalysts used for fuel production from sunlight. When the system is limited by charge transfer from the traps/catalyst layer and by detrapping, it is shown that only one capacitive peak is observable and is not recoverable in the return voltage scan. If the system is limited only by charge transfer and not by detrapping, two symmetric capacitive peaks can be observed in the cathodic and anodic directions. The model appears as a useful tool for the swift analysis of the electronic processes that limit fuel production.
\end{abstract}

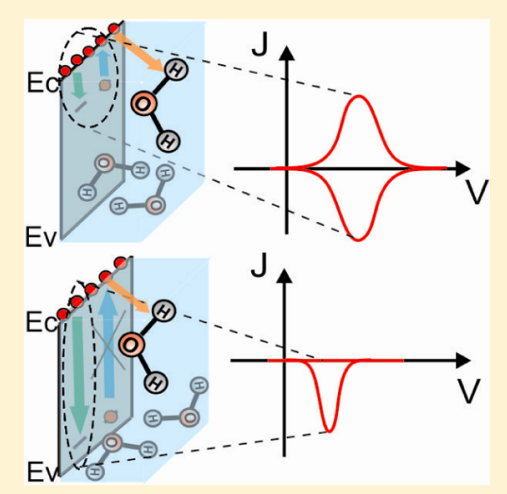

14 SECTION: Energy Conversion and Storage; Energy and Charge Transport

${ }_{\text {"i }} \mathrm{D}$ irect transformation of solar energy into chemical energy by hydrogen production through water splitting with 17 semiconductor materials in a photoelectrochemical cell 18 constitutes an attractive solution to our energy needs. However, 19 despite the intense efforts carried out in the last decades, no 20 single material has been identified satisfying all of the efficiency, 21 stability, and cost conditions needed for industrial deployment 22 of this technology. ${ }^{1-4}$

23 Hematite $\left(\alpha-\mathrm{Fe}_{2} \mathrm{O}_{3}\right)$ has emerged as a promising candi24 date $^{5-9}$ due to its abundance in the earth crust, visible light 25 absorption, and good stability in the harsh environmental 26 conditions needed for operation, although the obtained solar27 to-fuel efficiencies still remain low for commercial exploitation. 28 One of the main causes of the low performance of hematite is 29 related to the large overpotentials required for water oxidation 30 (around $500 \mathrm{mV}$ ), and surface treatments have proven to 31 enhance notably water splitting performances. ${ }^{10-12}$ It has been 32 suggested that the reasons for these large overpotentials are 33 related to sluggish hole transfer to the electrolyte e $^{13,14}$ and to the 34 existence of traps in the bulk and at the semiconductor/ 35 electrolyte interface, ${ }^{15-17}$ leading to high recombination. ${ }^{18,19}$ 36 Clearly, the separation of the different processes that constitute 37 the oxidative current and the identification of the main kinetic 38 bottlenecks are complex tasks. Therefore, the accurate 39 interpretation of the results provided by characterization 40 techniques constitutes a key tool to rationalize materials 41 development and device optimization. Recently, we have 42 proposed a simple physical model that allows the interpretation 43 of impedance spectroscopy (IS) spectra for water splitting 44 applications. ${ }^{20}$ In this model, we have considered a 45 monoenergetic level of surface states where both electron and 46 holes can recombine or transfer from/to the solution.
In the present study, we propose a complementary simple 47 model to predict the curves obtained by cyclic voltammetry 48 (CV). This characterization technique allows a quick test of the 49 faradic behavior associated with charge transfer and the 50 capacitive behavior associated with the separated modes of 51 carrier storage, which depend on the kinetics of the system at 52 stake. $^{21}$ Starting from the characteristic features of reported 53 voltammograms of hematite and related systems, we have 54 derived a model that is able to map the different kinetic 55 configurations of a system used for solar fuel applications. We 56 show that two types of peaks featuring charge storage in traps 57 can be observed when charge transfer from surface states is 58 kinetically limited. Those peaks allow characterization of the 59 degree of recombination at the surface states and are discussed 60 later on.

In Figure 1, we present the typical voltammetry plots $62 \mathrm{fl}$ obtained for an $\mathrm{Fe}_{2} \mathrm{O}_{3}$ sample synthetized by atmospheric 63 pressure chemical vapor deposition (APCVD). ${ }^{22,23}$ Figure 1a 64 shows the effect of a pretreatment in dark conditions at 65 different anodic potentials $V_{0}$ during $60 \mathrm{~s}$. The voltammetry 66 plots were recorded at a fixed scan rate $(500 \mathrm{mV} / \mathrm{s})$. This figure 67 displays a clear cathodic capacitive peak whose height increases 68 as the pretreatment anodic potential increases. A linear 69 dependence exists between the peak current and $V_{0}$, as can 70 be seen in Figure $1 \mathrm{~b}$. This peak can be attributed to the 71 charging of a monoenergetic level of surface states. It can be 72 remarked that at a low potential (here, $V_{0}=1.66 \mathrm{~V}$ versus 73

Received: March 13, 2013

Accepted: April 5, 2013 

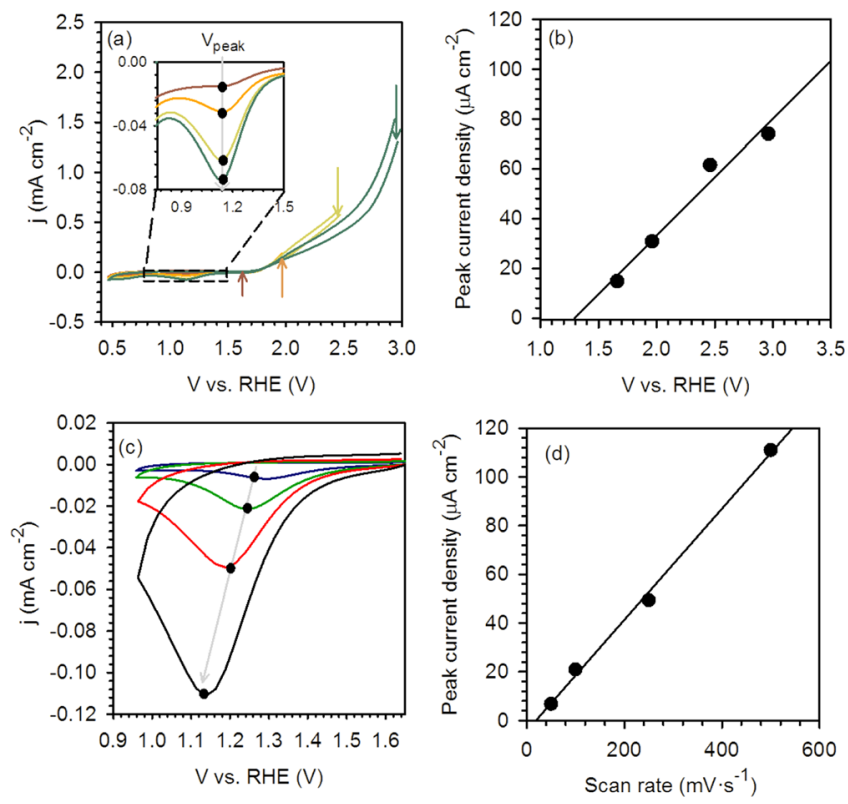

Figure 1. (a) Voltammogram of $\mathrm{Fe}_{2} \mathrm{O}_{3}$ in the dark after $60 \mathrm{~s}$ of pretreatment at different static potentials $V_{0}$ (indicated by arrows) at a fixed scan rate of $s=0.5 \mathrm{~V} \mathrm{~s}^{-1}$. Each capacitive peak occurs at the same voltage peak $V_{\text {peak }}=1.14 \mathrm{~V}$ versus RHE. Values of the capacitive peaks: $j_{1}=13 \mu \mathrm{A} \mathrm{cm}^{-2}, j_{2}=31 \mu \mathrm{A} \mathrm{cm}^{-2}, j_{3}=62 \mu \mathrm{A} \mathrm{cm}^{-2}$, and $j_{4}=74 \mu \mathrm{A}$ $\mathrm{cm}^{-2}$ (b) Peak current versus voltage extracted from (a). (c) Voltammogram of $\mathrm{Fe}_{2} \mathrm{O}_{3}$ in the dark after $60 \mathrm{~s}$ of pretreatment under 1 sun illumination at $V_{0}=1.66 \mathrm{~V}$ versus RHE and different scan rates $\left(50,100,250\right.$, and $\left.500 \mathrm{mV} \mathrm{s}^{-1}\right)$. Values of the capacitive peaks: $j_{1}^{\prime}$ $=7 \mu \mathrm{A} \mathrm{cm}^{-2}, j_{2}^{\prime}=21 \mu \mathrm{A} \mathrm{cm}^{-2}, j_{3}^{\prime}=49 \mu \mathrm{A} \mathrm{cm}^{-2}$, and $j_{4}^{\prime}=111 \mu \mathrm{A} \mathrm{cm}^{-2}$. (d) Peak current versus the scan rate extracted from (c).

74 RHE), the cathodic peak is almost nonexistent. This indicates 75 that traps are either totally filled by electrons and emptied by 76 hole trapping when applying a high positive bias or created by 77 oxidation of the hematite surface at higher voltage. As already 78 discussed elsewhere, in the case of hematite, traps are formed 79 by forcing the oxidation of the hematite surface by applying a 80 sufficiently positive voltage. ${ }^{24}$ After a pretreatment of one sun 81 illumination at the potential $V_{0}=1.66 \mathrm{~V}$ versus RHE, the 82 cathodic peak height increases with scan rate, Figure 1c. Note 83 that the voltammetry sweep is not performed under 84 illumination in order to avoid masking the trap capacitive 85 effects by the photocurrent. The voltage of the peak is shifted in 86 the cathodic direction when the scan rate increases, as indicated 87 by the gray arrow. The dependence of the cathodic peak 88 current with scan rate is linear, as shown in Figure 1d, 89 indicating that diffusion limitations do not exist for these 90 experiments. It should also be noticed that under dark 91 conditions at $V_{0}=1.66 \mathrm{~V}$ versus RHE and at a scan rate of $92500 \mathrm{mV} / \mathrm{s}$ (Figure 1a), the cathodic capacitive peak is hardly 93 visible, while under illumination, the peak is visible even at 50 $94 \mathrm{mV} / \mathrm{s}$.

95 The previous observations indicate that traps are created 96 chemically by oxidation of the hematite surface either by 97 imposing a higher positive bias or by illumination. It has been 98 suggested that these surface traps are $\mathrm{Fe}=\mathrm{O}$ intermediates and 99 the formation of these species by proton-coupled oxidation of 100 surface hydroxide species constitutes the first step of water 101 oxidation on hematite electrodes. ${ }^{16,25}$ In other materials, like $102 \mathrm{GaN}$ for instance, ${ }^{26}$ surface traps are mainly due to the 103 morphology of the material and are present in the dark and under illumination. Consequently, in the latter situation, the 104 voltammetry plots display a comparable capacitive peak for 105 both pretreatments. Additionally, it should also be noted that 106 no anodic peak is present in any of the plots represented in 107 Figure $1 \mathrm{a}$ and c. However, it has been recently remarked ${ }^{23}$ that 108 after deposition of an iridium-based catalyst to the hematite 109 surface, a quasi-symmetric peak can be observed at cathodic 110 potentials, as depicted by Figure 2. This peak, which is observed $111 \mathrm{f} 2$
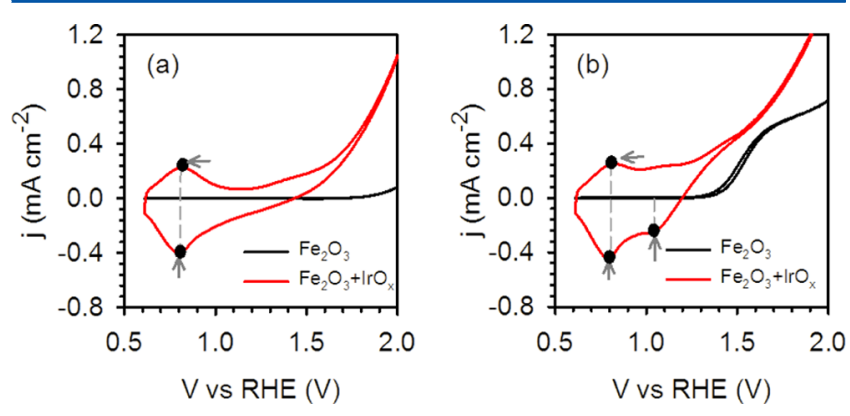

Figure 2. Cyclic voltammetry curves obtained in the dark (peak voltage: $V_{1}=0.8 \mathrm{~V}$ versus RHE; capacitive peaks: $j_{1}^{\text {cat }}=-0.4 \mathrm{~mA} \mathrm{~cm}{ }^{-2}$, $j_{1}^{\text {an }}=0.22 \mathrm{~mA} \mathrm{~cm}{ }^{-2}$ ) (a) and under illumination at $100 \mathrm{~mW} \mathrm{~cm}^{-2}$ (second peak voltage: $V_{2}=1.01 \mathrm{~V}$ versus RHE; cathodic peak: $j_{2}^{\text {cat }}=$ $-0.26 \mathrm{~mA} \mathrm{~cm}{ }^{-2}$ ) (b) for a reference $\mathrm{Fe}_{2} \mathrm{O}_{3}$ sample (black curve) and after electrodeposition of an $\mathrm{IrO}_{x}$ layer from a metallorganic [ $\mathrm{Cp} * \mathrm{Ir}$ $\left.\left(\mathrm{H}_{2} \mathrm{O}\right)_{3}\right]\left(\mathrm{SO}_{4}\right)$ precursor (red curve). Surface concentration of Ir on $\mathrm{Fe}_{2} \mathrm{O}_{3}: 3.9$ atom \%. Scan rate: $10 \mathrm{mV} \mathrm{s}^{-1}$. ${ }^{23}$

both in the dark and under illumination conditions, indicates 112 that $\mathrm{IrO}_{x}$ acts in a similar fashion as intrinsic surface states, by 113 capture and release of carriers from those states, which 114 facilitates charge transfer to solution. This capacitive feature 115 of $\mathrm{IrO}_{x}$ can be obviously related to the standard CV behavior of 116 redox species. More specifically, it has been ascribed to an 117 $\operatorname{Ir}(\mathrm{III}) / \operatorname{Ir}(\mathrm{IV})$ redox process, which involves a two-electron, 118 three-proton process. ${ }^{27,28}$ However, it is interesting to discuss 119 how the redox catalyst is electronically coupled to the 120 semiconductor film. Similar observations have been reported 121 with cobalt-phosphate $(\mathrm{Co}-\mathrm{Pi})$ catalyst layers covering 122 hematite electrodes. ${ }^{15}$

In the following, we present a simple model that allows 124 prediction of the voltammetry plots in the presence of a 125 monoenergetic level of surface states with a pretreatment done 126 under illumination at a voltage above the onset voltage. The 127 model, which is based on a previous model developed by 128 Bisquert, $^{29}$ allows prediction of the voltammetry patterns 129 reported in the present study and, in general, in the 130 measurement of solar water splitting semiconductor films. 131

We consider a thin and homogeneous semiconductor film of 132 length $d$ with a density of $N_{\mathrm{t}}$ monoenergetic traps per unit of 133 volume, as shown in Figure 3. We term $n$ and $f$ as the density of $134 \mathrm{f} 3$ electrons in the conduction band and the traps occupation 135 probability, respectively. $n_{0}$ and $f_{0}$ are the same respective 136 quantities taken at equilibrium. The detailed calculation of $f_{0}$ is 137 given in the Supporting Information (SI). Because the 138 distribution of carriers in the semiconductor layer is assumed 139 to be homogeneous, $n$ only depends on time and is governed 140 by the applied voltage $V$

$$
n(t)=n_{0} \exp \left(-\frac{q V(t)}{k_{\mathrm{B}} T}\right)
$$

where $k_{\mathrm{B}} T$ is the thermal energy. 


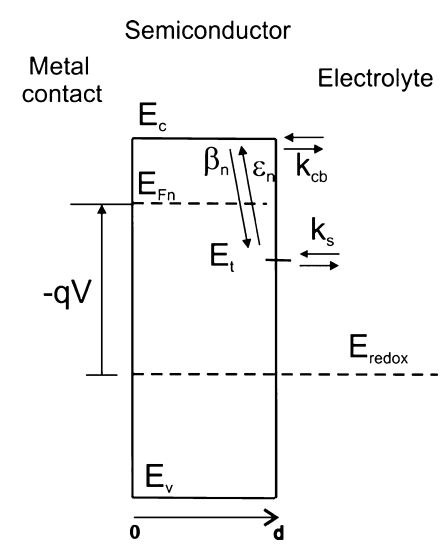

Figure 3. Scheme of the kinetics of the processes occurring at the interface of the semiconductor/solution. $d$ is the thickness of the semiconductor layer. The processes are trapping of electrons from the conduction band $\left(\beta_{\mathrm{n}}\right)$ and detrapping $\left(\varepsilon_{\mathrm{n}}\right)$ and charge transfer of electrons from the traps $\left(k_{\mathrm{s}}\right)$ and from the conduction band $\left(k_{\mathrm{cb}}\right)$.

144 It is well-known that the presence of an electric field in the 145 space charge region improves charge separation in the active 146 layer, which can enhance the fuel production efficiency. ${ }^{12}$ In the 147 model below, we address the question of trap occupation and 148 charge transfer from the surface states, depending on the 149 homogeneous electrode potential. The same model could be 150 formulated for a surface Schottky barrier with simple 151 modifications, but these considerations are beyond the scope 152 of this work. Henceforth, for the sake of simplicity, the 153 influence of the electric field at the interface of the electrolyte/ 154 semiconductor is neglected.

155 Initially, before the voltammetry scan, a pretreatment is done 156 in order to fill the traps with holes at a fixed voltage $V_{0}$ more 157 positive than the current onset voltage. Therefore, when the 158 voltammetry sweep starts, that is, at $t=0$, we consider that $159 f(t=0)=0$. For a more complete treatment, $f(t=0)$ should be 160 calculated by taking into account both electron and hole 161 recombination and charge transfer from traps. However, for 162 simplicity, the dynamics of holes are not included in Figure 3. 163 For the interested reader, such calculations can be found in ref 16420 for the technique of IS.

165 During the cyclic voltammetry scan, that is, at time $t>0$, the 166 semiconductor is in the dark. Two types of processes are 167 considered in this case, (i) charge transfer from the conduction 168 band (kinetic constant $k_{\mathrm{cb}}$ ) and from the traps $\left(k_{\mathrm{s}}\right)$ and (ii) 169 electron trapping $\left(\beta_{\mathrm{n}}\right) /$ detrapping $\left(\varepsilon_{\mathrm{n}}\right)$. We aim to calculate the 170 current density $j_{n}$, and we use the usual boundary conditions, eq 1711 at the left metal/semiconductor contact, and at the right, the 172 semiconductor/electrolyte contact is considered to be a 173 blocking layer (i.e., $j_{n}(d, t)=0$ ). The voltage that appears in 174 eq 1 varies with time as

$\left\{\begin{array}{l}V(t \leq \lambda)=V_{0}-s t \\ V(t \geq \lambda)=V_{0}-2 s \lambda+s t\end{array}\right.$

176 where $s$ is the scan rate and $\lambda$ the period of the voltage sweep. 177 Integration of the continuity equation for electrons in the 178 conduction band and the master equation for electrons in the 179 traps along the homogeneous layer of length $d$ leads to

$$
\frac{\partial n}{\partial t}=-\frac{1}{q d} j_{n}(0)-\beta_{\mathrm{n}}(1-f) n N_{\mathrm{t}}+\varepsilon_{\mathrm{n}} f N_{\mathrm{t}}-k_{\mathrm{cb}}\left(n-n_{0}\right)
$$

$$
\frac{\partial f}{\partial t}+\varepsilon_{\mathrm{n}}+k_{\mathrm{s}}\left(1-f_{0}\right)=\left(\beta_{\mathrm{n}} n+\varepsilon_{\mathrm{n}}+k_{\mathrm{s}}\right)(1-f)
$$

We shall introduce some useful quantities, namely, the 182 equilibrium chemical capacitances ${ }^{30,31}$ associated with each 183 charge storage mode. We define the well-known chemical 184 capacitance for electrons in the conduction band

$$
C_{\mu}^{\mathrm{cb}}=q d\left|\frac{\partial n}{\partial V}\right|=\frac{q^{2} d}{k T} n
$$

and the equilibrium chemical capacitance of the traps ${ }^{32-34}$

$$
C_{\mu \mathrm{eq}}^{s s}=q d\left|\frac{\partial f}{\partial V}\right| N_{\mathrm{t}}
$$

Note that the latter capacitance relies on the knowledge of $f, 189$ which relies on the resolution of eq 4. Equations 2-4 provide 190 the general form of the voltammetric current

$$
\left\{\begin{array}{l}
j_{n}(t \leq \lambda)=j_{\text {res }}-j_{\text {cap }} \\
j_{n}(t \geq \lambda)=j_{\text {res }}+j_{\text {cap }}
\end{array}\right.
$$

where $j_{\text {res }}$ is the resistive current density (i.e., faradaic current) 193

$$
j_{\text {res }}=-q d\left[k_{\mathrm{cb}}+(1-B) \varepsilon_{\mathrm{n}} \frac{N_{\mathrm{t}}}{n_{0}} f_{0}\right] \times\left(n-n_{0}\right)
$$

with

$$
B=1-\frac{k_{\mathrm{s}}}{\beta_{\mathrm{n}} n+\varepsilon_{\mathrm{n}}+k_{\mathrm{s}}}
$$

$j_{\text {cap }}$ is the capacitive current density given by

$$
j_{\text {cap }}=\left[C_{\mu}^{\mathrm{cb}}+B C_{\mu \mathrm{eq}}^{\mathrm{ss}}\right] \times s
$$

The first term on the right-hand side of eq 8 corresponds to the 199 charge transfer from the conduction band, while the second 200 term has already been associated through IS to the trapping/ 201 detrapping process and charge transfer from the traps. ${ }^{29} 202$

The general solution of the above set of equations can be 203 obtained numerically, but it is useful to distinguish between 204 different physical cases. In the model of Figure 3, we will 205 consider that charge transfer from the traps is slow enough 206 compared to the velocity of trap charging so that a capacitance 207 peak can be observed. This implies that $k_{\mathrm{s}} \ll q s / k_{\mathrm{B}} T$. Note that 208 in the case where charge transfer from the traps is zero $\left(k_{\mathrm{s}}=0\right), 209$ the following treatment can also be applied for the bulk of the 210 semiconductor if it is homogeneous enough so that eqs 3 and 4211 remain valid. However, this latter case is not desired for fuel 212 production because it would imply high onset voltages. Besides, 213 as indicated by the experimental CV patterns of Figures 1 and 214 2 , it is important to distinguish two types of behaviors; either 215 trapping/detrapping is fast enough in comparison with the trap 216 charging velocity, that is, $\varepsilon_{\mathrm{n}} \gg q s / k_{\mathrm{B}} T$ or inversely.

In the case where trapping/detrapping is very fast, traps are 218 in equilibrium with the semiconductor conduction band, while 219 in the second case, electrons accumulate in the traps and are 220 therefore subject to much higher recombination. In the first 221 case, the onset voltage should therefore be lower than that in 222 the second one. Let us now examine both cases in terms of the 223 voltammetry pattern and relate them to the experimental cases 224 depicted by Figures 1 and 2 . 


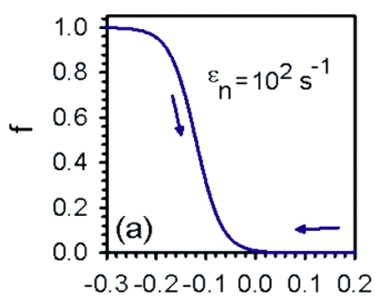

Potential (V)

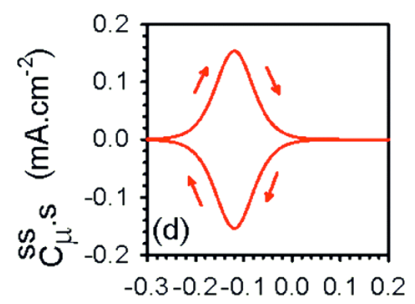

Potential (V)

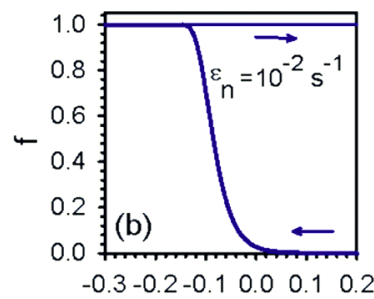

Potential (V)

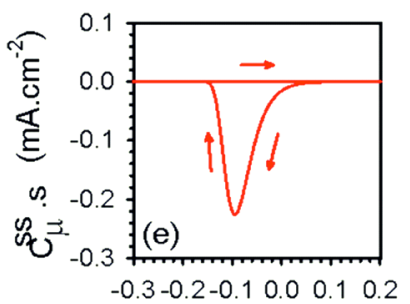

Potential (V)

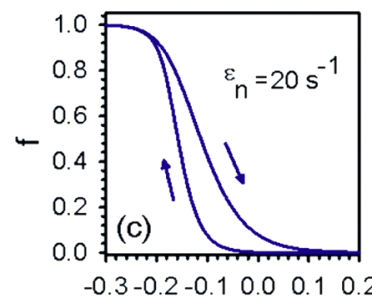

Potential (V)

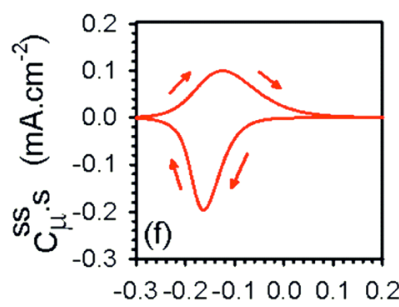

Potential (V)

Figure 4. Trap occupation probability for a system limited by charge transfer and (a) when detrapping is fast with respect to the charging velocity (i.e., traps are in equilibrium with the conduction band), (b) when detrapping is slow (i.e., high recombination), and (c) for an intermediate case. (d-f) Trap capacitive current derived from the respective occupation probability of (a-c). Simulation parameters: $k T=26 \mathrm{meV}, d=1 \mathrm{~nm}, N_{\mathrm{t}}=10^{21}$ $\mathrm{cm}^{-3}, \beta_{\mathrm{n}} n_{0}=1 \mathrm{~s}^{-1}, k_{\mathrm{s}}=10^{-4} \mathrm{~s}^{-1}, s=1 \mathrm{~V} \cdot \mathrm{s}^{-1}$.

226 In Figure 4, we give examples of representation of the trap 227 occupation probability $f$ for a system limited by charge transfer. 228 We illustrate three cases, when detrapping is fast, depicted in 229 Figure 4a, when detrapping is slow, shown in Figure 4b, and an 230 intermediary case represented in Figure 4c. The corresponding 231 capacitive currents $\left(s C_{\mu}^{\mathrm{ss}}\right)$ from the traps are given in Figure $2324 \mathrm{~d}-\mathrm{f}$. In Figure 5, we give the total voltammetric current 233 obtained from the numerical resolution of eqs 3 and 4 in 234 different cases discussed below.

235 We now discuss the influence of the trap kinetics on the 236 voltammetry patterns for semiconductors used for water 237 splitting applications. We focus on the possible shapes of the $238 \mathrm{CV}$ patterns obtained in the framework of our model. We then 239 compare them to both experimental examples of Figures 1 and 2402 , which are representative of the main voltammetry plots that 241 can be found in the literature for this type of system.

242 When the voltammetry scan is carried out in the cathodic 243 direction, the electron Fermi level is shifted upward, and traps 244 (initially filled with holes because of the pretreatment) are filled 245 with electrons while the conduction band is filled at more 246 cathodic voltage. The voltammetry pattern therefore displays a 247 first cathodic capacitive peak induced by the filling of the traps 248 by electrons followed either by the faradic current from the 249 conduction band (Figure $5 \mathrm{~b}$ and $\mathrm{d}$ ) or by the capacitive current 250 (Figures $5 \mathrm{a}$ and $\mathrm{c}$ ). In the anodic direction, because charge 251 transfer from the traps is slow with respect to the trap charging 252 velocity, traps are emptied relatively according to the 253 detrapping rate.

254 In the case where detrapping is very slow (Figure 5a and b) 255 with respect to the trap charging velocity, charges accumulate in 256 the traps in the cathodic direction. Note that in this case, the 257 voltage of the cathodic peak depends on the scan rate and is 258 shifted toward the cathodic direction as the scan rate increases. 259 The mathematical demonstration of this result is given in the 260 SI. In the anodic direction, because detrapping is very slow, the 261 charges accumulated in the traps are extracted much slower 262 than they were injected in the cathodic direction. For this 263 reason, the occupation probability is almost constant and 264 maximum in the anodic direction (Figure 4b). As a
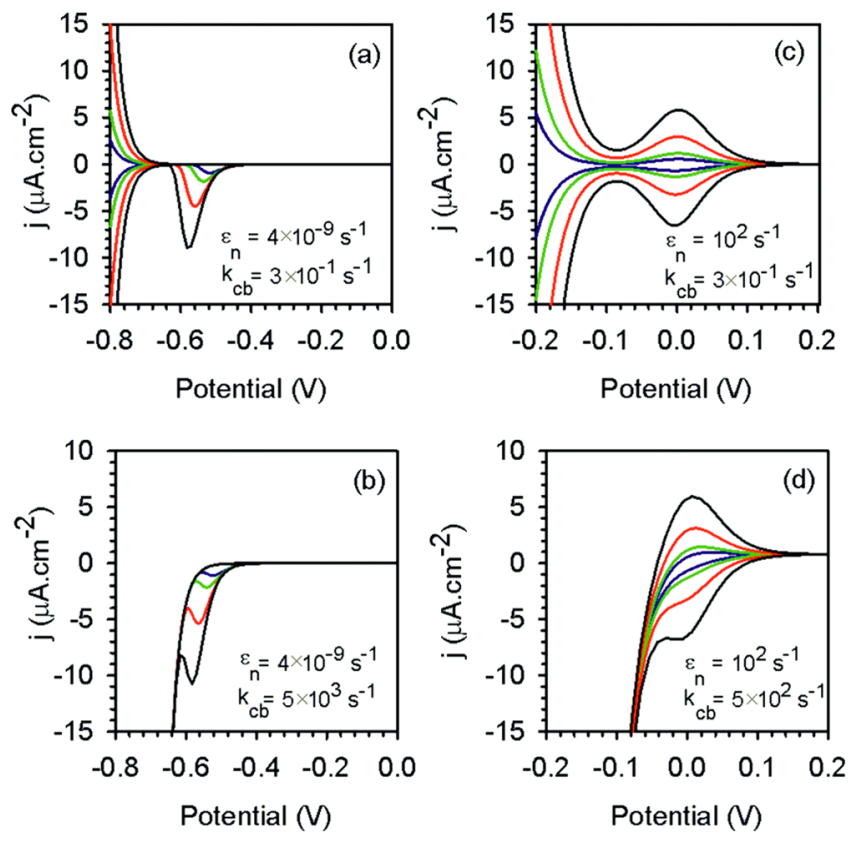

Figure 5. Voltammetric plots obtained by solving numerically eqs 3 and 4 under the condition that charge transfer is a limiting kinetic factor. Four different cases are computed where detrapping $\left(\varepsilon_{\mathrm{n}}=\beta_{\mathrm{n}} n_{0}\right)$ and charge transfer from the conduction band $\left(k_{\mathrm{cb}}\right)$ are analyzed. The general parameters of this simulation are $k T=26 \mathrm{meV}, d=1 \mathrm{~nm}, N_{\mathrm{t}}=$ $8 \times 10^{19} \mathrm{~cm}^{-3}, k_{\mathrm{s}}=10^{-2} \mathrm{~s}^{-1}$. For each figure, the blue plot corresponds to the scan rate $50 \mathrm{mV} \cdot \mathrm{s}^{-1}$, the green plot to $100 \mathrm{mV} \cdot \mathrm{s}^{-1}$, the red plot to $250 \mathrm{mV} \cdot \mathrm{s}^{-1}$, and the black one to $500 \mathrm{mV} \cdot \mathrm{s}^{-1}$.

consequence, no anodic peak can be observed. Experimentally, 265 this latter case is depicted by Figure 1 for bare hematite. In this 266 case, traps are formed by oxidation of the hematite surface. The 267 shape of the capacitive peak that appears after illumination of 268 the sample indicates that not only is the charge transfer from 269 the traps slow, but also, the detrapping process is sluggish. 270 Thereby, traps actuate as recombination centers and limit the 271 oxygen evolution. The same behavior has been reported in the 272 
273 case of $\mathrm{GaN}^{26}$ Nonetheless, in this case, surface states are 274 intrinsic and display a capacitive peak in both dark and 275 illumination conditions, though the peak intensity is higher 276 under illumination compared to that under dark conditions. 277 This is due to the fact that in the dark, those surface states are 278 initially partially filled with electrons, while under illumination, 279 traps are emptied by hole trapping, which allows for higher 280 charge accumulation during the voltammetry sweep.

281 In the case where both trapping and detrapping are fast 282 compared to the trap charging velocity (Figure $5 \mathrm{c}$ and $\mathrm{d}$ ), 283 charge accumulation is reversible, and the occupation 284 probability is equal in both the anodic and cathodic directions 285 (Figure 4a). Hence, the trap capacitance is the same in both 286 directions, and the trap capacitive current is symmetric and 287 proportional to the scan rate (see the SI for more information 288 on the capacitance peak voltage). This situation corresponds to 289 the experimental case depicted by Figure 2 . When the hematite 290 surface is treated with an iridium-based catalyst, two quasi291 symmetric peaks, cathodically shifted with respect to the bare 292 hematite peak, appear under both dark and illumination 293 conditions. This indicates that $\mathrm{IrO}_{x}$ highly reversible oxidation 294 states act similarly to intrinsic surface states and that detrapping 295 is much easier in this case. Consequently, the $\mathrm{IrO}_{x}$ catalyst 296 enhances the detrapping process and decreases recombination. 297 For this reason, higher currents and lower onset voltages can be 298 achieved. $^{35}$ It has also been shown by IS that $\mathrm{IrO}_{x}$ enhances 299 charge transfer. ${ }^{23}$ Additionally, it should be remarked that a 300 second cathodic peak appears under illumination. We believe 301 that this peak corresponds to the surface states created by 302 oxidation of the hematite surface, as already observed for bare 303 hematite. It should also be remarked that in other recent 304 studies, the capacitive response of surface states has been found 305 to be reduced in the presence of $\mathrm{Ga}_{2} \mathrm{O}_{3}$ and $\mathrm{Al}_{2} \mathrm{O}_{3}$ 306 overlayers. $^{36,37}$ It has been suggested that 13-group oxide 307 overlayers passivate surface states by releasing lattice strain of 308 the hematite layer. On the other hand, the deposition of cobalt309 based catalyst layers also leads to a decrease of surface state 310 capacitance by accelerating the charge-transfer rate from surface 311 states. ${ }^{38}$ It should be mentioned that these previous studies 312 were carried out under illumination, and consequently, the 313 capacitive response of the electrodes was partially masked by 314 the photocurrent, in contrast with our present study in dark 315 conditions.

316 In conclusion, we have shown that when charge transfer is 317 very slow, at least one capacitive peak can be observed in the 318 voltammograms corresponding to the system at stake. The 319 presence of one capacitive peak only in the cathodic direction 320 (i.e., without the corresponding symmetric anodic peak) is a 321 feature of a system exhibiting high recombination. In this case, 322 the peak voltage is shifted in the cathodic direction as the scan 323 rate increases. On the contrary, two symmetric peaks 324 characterize a system with trap states in equilibrium with the 325 conduction band and display lower recombination compared to 326 the previous case. In this case, both peaks increase propor327 tionally to the scan rate, and the peak voltage is constant with 328 scan rate. A system that is characterized by a good charge 329 transfer from traps does not display any capacitive peak.

\section{ASSOCIATED CONTENT}

\section{S Supporting Information}

332 The detailed calculation of the trap occupation probability at 333 equilibrium and the capacitance peak voltage observed in the $334 \mathrm{CV}$ patterns in both cases of slow and fast trapping/detrapping with respect to the trap charging velocity. This material is 335 available free of charge via the Internet at http://pubs.acs.org. 336

\section{AUTHOR INFORMATION}

\section{Corresponding Author}

*E-mail: bisquert@uji.es.

Notes

The authors declare no competing financial interest.

\section{ACKNOWLEDGMENTS}

The research leading to these results is supported by 343 Universitat Jaume I Project P1·1B2011-50.

\section{REFERENCES}

(1) Bolts, J.; Wrighton, M. Correlation of Photocurrent-Voltage 346 Curves with Flat-Band Potential Stable Photoelectrodes for the 347 Photoelectrolysis of Water. J. Phys. Chem. 1976, 80, 2641-2645. 348

(2) Nozik, A. J. Photoelectrochemistry: Applications to Solar Energy 349 Conversion. Annu. Rev. Phys. Chem. 1978, 29, 189.

(3) Butler, M. A.; Ginley, D. S. Principles of Photoelectrochemical, 351 Solar Energy Conversion. J. Mater. Sci. 1980, 15, 1-19.

(4) Walter, M. G.; Warren, E. L.; McKone, J. R.; Boettcher, S. W.; 353 Mi, Q. X.; Santori, E. A.; Lewis, N. S. Solar Water Splitting Cells. 354 Chem. Rev. 2010, 110, 6446-6473.

(5) Kennedy, J. H. Photooxidation of Water at $\alpha-\mathrm{Fe}_{2} \mathrm{O}_{3}$ Electrodes. J. 356 Electrochem. Soc. 1978, 125, 709.

(6) Murphy, A.; Barnes, P. Randeniya, L. Plumb, I. Grey, I.; Horne, 358 M.; Glasscock, J. Efficiency of Solar Water Splitting Using Semi- 359 conductor Electrodes. Int. J. Hydrogen Energy 2006, 31, 1999-2017. 360 (7) Dare-Edwards, M. P.; Goodenough, J. B.; Hamnett, A.; 361 Trevellick, P. R. Electrochemistry and Photoelectrochemistry of 362 Iron(III) Oxide. J. Chem. Soc., Faraday Trans. 1 1983, 79, 2027-2041. 363

(8) Katz, M. J.; Riha, S. C.; Jeong, N. C.; Martinson, A. B. F.; Farha, 364 O. K.; Hupp, J. T. Toward Solar Fuels: Water Splitting with Sunlight 365 and Rust? Coord. Chem. Rev. 2012, 256, 2521-2529.

(9) Peter, L. M. Energetics and Kinetics of Light-Driven Oxygen 367 Evolution at Semiconductor Electrodes: The Example of Hematite. J. 368 Solid State Electrochem. 2012, 17, 315-326.

(10) Barroso, M.; Cowan, A. J.; Pendlebury, S. R.; Gratzel, M.; Klug, 370 D. R.; Durrant, J. R. The Role of Cobalt Phosphate in Enhancing the 371 Photocatalytic Activity of $\alpha$ - $\mathrm{Fe}_{2} \mathrm{O}_{3}$ toward Water Oxidation. J. Am. 372 Chem. Soc. 2011, 133, 14868-14871.

373

(11) Zhong, D. K.; Choi, S.; Gamelin, D. R. Near-Complete 374 Suppression of Surface Recombination in Solar Photoelectrolysis by 375 "Co-Pi” Catalyst-Modified W:BiVO4. J. Am. Chem. Soc. 2011, 133, 376 18370-18377.

(12) Barroso, M.; Mesa, C. A.; Pendlebury, S. R.; Cowan, A. J.; 378 Hisatomi, T.; Sivula, K.; Gratzel, M.; Klug, D. R.; Durrant, J. R. 379 Dynamics of Photogenerated Holes in Surface Modified $\alpha-\mathrm{Fe}_{2} \mathrm{O}_{3} 380$ Photoanodes for Solar Water Splitting. Proc. Natl. Acad. Sci. U.S.A. 381 2012, 109, 15640-15645.

382

(13) Wijayanthaa, K. G. U.; Saremi-Yarahmadia, S.; Peter, L. M. 383 Kinetics of Oxygen Evolution at $\alpha$ - $\mathrm{Fe}_{2} \mathrm{O}_{3}$ Photoanodes: A Study by 384 Photoelectrochemical Impedance Spectroscopy. Phys. Chem. Chem. 385 Phys. 2010, 13, 5264-5270.

(14) Peter, L. M.; Wijayantha, K. G. U.; Tahir, A. A. Kinetics of 387 Light-Driven Oxygen Evolution at $\alpha-\mathrm{Fe}_{2} \mathrm{O}_{3}$ Electrodes. Faraday 388 Discuss. 2012, 155, 309-322.

(15) Klahr, B.; Gimenez, S.; Fabregat-Santiago, F.; Bisquert, J.; 390 Hamann, T. W. Photoelectrochemical and Impedance Spectroscopic 391 Investigation of Water Oxidation with $\mathrm{Co}-\mathrm{Pi}$-Coated Hematite 392 Electrodes. J. Am. Chem. Soc. 2012, 134, 16693-16700.

(16) Klahr, B.; Gimenez, S.; Fabregat-Santiago, F.; Bisquert, J.; 394 Hamann, T. W. Electrochemical and Photoelectrochemical Inves- 395 tigation of Water Oxidation with Hematite Electrodes. Energy. Environ. 396 Sci. 2012, 5, 7626. 
398 (17) Braun, A.; Sivula, K.; Bora, D. K.; Zhu, J.; Zhang, L.; Grätzel, M.; 399 Guo, J.; Constable, E. C. Direct Observation of Two Electron Holes in 400 a Hematite Photo-Anode During Photo-Electrochemical Water 401 Splitting. J. Phys. Chem. C 2012, 116, 16870-16875.

402 (18) Vanmaekelbergh, D.; Cardon, F. Calculation of the Electrical 403 Impedance Associated with the Surface Recombination of Free 404 Carriers at an Illuminated Semiconductor/Electrolyte Interface. J. 405 Phys. D: Appl. Phys. 1986, 19, 643.

406 (19) Ponomarev, E. A.; Peter, L. M. A Comparison of Intensity 407 Modulated Photocurrent Spectroscopy and Photoelectrochemical 408 Impedance Spectroscopy in a Study of Photoelectrochemical Hydro409 gen Evolution at p-InP. J. Electrochem. Chem. 1995, 397, 45-52.

410 (20) Bertoluzzi, L.; Bisquert, J. Equivalent Circuit of Electrons and 411 Holes in Thin Semiconductor Films for Photoelectrochemical Water 412 Splitting Applications. J. Phys. Chem. Lett. 2012, 3, 2517-2522.

413 (21) Fabregat-Santiago, F.; Mora-Seró, I.; Garcia-Belmonte, G.; 414 Bisquert, J. Cyclic Voltammetry Studies of Nanoporous Semi415 conductor Electrodes. Models and Application to Nanocrystalline $416 \mathrm{TiO}_{2}$ in Aqueous Electrolyte. J. Phys. Chem. B 2003, 107, 758-769.

417 (22) Kay, A.; Cesar, I.; Grätzel, M. New Benchmark for Water 418 Photooxidation by Nanostructured $\alpha-\mathrm{Fe}_{2} \mathrm{O}_{3}$ Films. J. Am. Chem. Soc. 419 2006, 128, 15714-15721.

420 (23) Badia-Bou, L.; Mas Marzá, E.; Rodenas, P.; Barea, E. M.; 421 Fabregat-Santiago, F.; Gimenez, S.; Peris, E. V.; Bisquert, J. Water 422 Oxidation at Hematite Photoelectrodes with an Iridium Based 423 Catalyst. J. Phys. Chem. C 2013, 117, 3826-3833.

424 (24) Jaegermann, W. The Semiconductor/Electrolyte Interface: A 425 Surface Science Approach. In Modern Aspects of Electrochemistry; 426 White, R. E., Ed.; Plenum Press: New York, 1996; No. 30.

427 (25) Hellman, A.; Pala, R. G. S. A First-Principles Study of Photo428 Induced Water-Splitting on $\mathrm{Fe}_{2} \mathrm{O}_{3}$. J. Phys. Chem. C 2011, 115, 429 12901-12907.

430 (26) Schäfer, S.; Koch, A. H. R.; Cavallini, A.; Stutzmann, M.; Sharp, 431 I. D. Charge Transfer across the n-Type GaN-Electrolyte Interface. J. 432 Phys. Chem. C 2012, 116, 22281-22286.

433 (27) Blakemore, J. D.; Schley, N. D.; Olack, G. W.; Incarvito, C. D.; 434 Brudvig, G. W.; Crabtree, R. H. Anodic Deposition of a Robust 435 Iridium-Based Water-Oxidation Catalyst from Organometallic Pre436 cursors. Chem. Sci. 2011, 2, 94-98.

437 (28) Burke, L. D.; Whelan, D. P. A New Interpretation of the Charge 438 Storage and Electrical-Conductivity Behavior of Hydrous Iridium 439 Oxide. J. Electroanal. Chem. 1981, 124, 333-337.

440 (29) Bisquert, J. Theory of the Impedance of Charge Transfer via 441 Surface States in Dye-Sensitized Solar Cells. J. Electrochem. Chem. 442 2010, 646, 43-51.

443 (30) Bisquert, J. Chemical Capacitance of Nanostructured Semi444 conductors: Its Origin and Significance for Heterogeneous Solar Cells. 445 Phys. Chem. Chem. Phys. 2003, 5, 5360-5364.

446 (31) Bisquert, J. Beyond the Quasi-Static Approximation: Impedance 447 and Capacitance of an Exponential Distribution of Traps. Phys. Rev. B 448 2008, 77, 235203.

449 (32) Frese, K. W.; Morrison, S. R. Electrochemical Measurements of 450 Interface States at the GaAs/Oxide Interface. J. Electrochem. Soc. 1979, 451 126, 1235-1241.

452 (33) Dare-Edwards, M. P.; Hamnett, A.; Trevellick, P. R. Alternating453 Current Techniques in Semiconductor Electrochemistry. J. Chem. Soc., 454 Faraday Trans. 1 1983, 79, 2111-2124.

455 (34) Allongue, P.; Cachet, H. I-V Curve and Surface State 456 Capacitance at Illuminated Semiconductor/Liquid Contacts. J. Electro457 chem. Chem. 1984, 176, 369-375.

458 (35) Tilley, S. D.; Cornuz, M.; Sivula, K.; Gratzel, M. Light-Induced 459 Water Splitting with Hematite: Improved Nanostructure and Iridium 460 Oxide Catalysis. Angew. Chem., Int. Ed. 2010, 49, 6405-6408.

461 (36) Le Formal, F.; Sivula, K.; Grätzel, M. The Transient 462 Photocurrent and Photovoltage Behavior of a Hematite Photoanode 463 under Working Conditions and the Influence of Surface Treatments. J. 464 Phys. Chem. C 2012, 116, 26707-26720.

465 (37) Hisatomi, T.; Le Formal, F.; Cornuz, M.; Brillet, J.; Tetreault, 466 N.; Sivula, K.; Gratzel, M. Cathodic Shift in Onset Potential of Solar
Oxygen Evolution on Hematite by 13-Group Oxide Overlayers. Energy 467 Environ. Sci. 2011, 4, 2512-2515.

(38) Riha, S. C.; Klahr, B. M.; Tyo, E. C.; Seifert, S.; Vajda, S.; Pellin, 469 M. J.; Hamann, T. W.; Martinson, A. B. F. Atomic Layer Deposition of 470 a Submonolayer Catalyst for the Enhanced Photoelectrochemical 471 Performance of Water Oxidation with Hematite. ACS Nano 2013, 7, 472 2396-2405. 